Théologiques

Théologiques

\title{
Les médiations de l'immédiateté. L’individualisme communautaire des assemblées pentecôtistes
}

\section{Laurent Amiotte-Suchet}

Volume 16, numéro 2, 2008

L’individualisation de la relation religieuse

URI : https://id.erudit.org/iderudit/001717ar

DOI : https://doi.org/10.7202/001717ar

Aller au sommaire du numéro

\section{Éditeur(s)}

Faculté de théologie et de sciences des religions, Université de Montréal

\section{ISSN}

1188-7109 (imprimé)

1492-1413 (numérique)

Découvrir la revue

\section{Citer cet article}

Amiotte-Suchet, L. (2008). Les médiations de l'immédiateté. L'individualisme communautaire des assemblées pentecôtistes. Théologiques, 16(2), 105-121. https://doi.org/10.7202/001717ar
Résumé de l'article

Lorsqu'il est amené à témoigner de sa conversion, le membre d'une assemblée pentecôtiste insiste avant tout sur la dimension personnelle de son choix de vie et sur les manifestations divines qui l'ont encouragé et qui l'encouragent encore chaque jour dans cette voie. Avoir fait l'expérience personnelle de l'agir divin est un élément constitutif de tout récit de conversion. Cet article, basé sur des recherches doctorales menées auprès de deux assemblées pentecôtistes de l'Est de la France, cherche à mettre en évidence que cette relation personnelle et intime entre le fidèle et son Créateur se construit dans le cadre communautaire : à travers l'harmonisation des récits de conversion et la structuration des réunions de prières assurant par les manifestations charismatiques la mise en présence de l'Esprit saint au sein de la communauté des fidèles. C'est en effet sur une validation communautaire que reposent en partie les convictions individuelles. 


\title{
Les médiations de l'immédiateté.
}

\author{
L'individualisme communautaire
}

des assemblées pentecôtistes

\author{
Laurent AMIOTTE-SUCHET" \\ Observatoire des religions en Suisse \\ Université de Lausanne
}

\section{Introduction}

«Dieu est engagé à se manifester, vous ne devez pas hésiter à le lui rappeler ", déclarait un jour le pasteur pentecôtiste à son assemblée. Force est de constater que ce type d'affirmation publique n'est pas monnaie courante dans les Églises protestantes luthéro-réformées, ni même dans les assemblées protestantes évangéliques. Le pasteur évoque ici l'idée d'une relation contractuelle, fondée sur l'alliance entre Dieu et son peuple. Dans la relation qui s'établit entre le fidèle et son Créateur, chacun doit en somme remplir sa part du marché. Puisque le Christ est mort en croix pour racheter les péchés de l'humanité, le nouveau venu au sein de l'assemblée apprend à considérer qu'il est de son devoir de sacrifier à son tour son existence pour préparer le retour de son Créateur, l'avènement du Royaume. Mais dans le même temps, Christ a promis qu'il doterait ceux qui proclameront partout sa Bonne Nouvelle d'une force nouvelle: la puissance de l'Esprit ${ }^{1}$. Si, pour

* Laurent Amiotte-Suchet est maître assistant à la Faculté de théologie et de sciences des religions de l'Université de Lausanne où il enseigne la sociologie des religions. Il est rattaché à l'Observatoire des religions en Suisse (ORS) dirigé par le sociologue Jörg Stolz. Il est également impliqué dans le projet de recherche « National Congregation Study in Switzerland»(NCSS) financé par le Fond national suisse de la recherche scientifique. Sa thèse doctorale, soutenue à l'École Pratique des Hautes Études en décembre 2006 s'intitule: "Pratiques pentecôtistes et dévotion mariale: analyse comparée des modes de mise en présence du divin. »

1. Voir Ac 1,8. Sur les expériences de la Pentecôte comme accomplissement de la promesse, voir Ac 2,1-9; 10,4-46 et 19,5-6. 
les membres des assemblées pentecôtistes, la conversion porte la marque d'un certain renoncement aux plaisirs du monde au nom d'un idéal à venir, elle porte également la marque de l'immédiateté de l'agir divin, force protectrice et revivifiante qui se pense autant comme une récompense de la conversion que comme une confirmation chaque jour renouvelée de l'authenticité de cette dernière.

\section{Terrains d'enquête: deux assemblées pentecôtistes de l'Est de la France}

Ce texte prend pour objet deux assemblées pentecôtistes de l'Est de la France que nous avons eu l'occasion de suivre durant près de quatre années dans le cadre de nos recherches doctorales (Amiotte-Suchet 2006). Ces deux assemblées - l'Église évangélique de Pentecôte de Besançon ${ }^{2}$ (EEP) et la Mission du Plein Évangile. La Porte Ouverte chrétienne de Mulhouse ${ }^{3}$ (POC) - avaient, à l'époque de nos investigations ethnographiques, un certain nombre de points communs. Assemblées regroupant chaque semaine un nombre important de fidèles pour le culte dominical, elles étaient toutes deux dirigées par des leaders ayant choisi de ne pas rester sous la tutelle des Assemblées de Dieu (ADD), la principale dénomination pentecôtiste en France. Les deux hommes - René Kennel pour l'EEP et Jean Peterschmitt pour la POC - étaient issus des milieux mennonites et s'étaient tous deux tournés vers le pentecôtisme dans les années 1960, à la suite des évangélisations tsiganes. Après des heures parfois difficiles et des prises de responsabilités progressives, René et Jean avaient respectivement construit une œuvre évangélique et pentecôtiste, formant à leur tour des pasteurs pour qu'ils puissent créer de nouvelles assemblées. Si René Kennel avait choisi de créer sa propre fédération pour y rassembler les assemblées nées de son ministère ${ }^{4}$, Jean Peterschmitt, lui, à la suite des expériences

2. Nos investigations à l'Église évangélique de Pentecôte de Besançon ont débuté en 1998, dans cadre d'une maîtrise universitaire. L'assemblée bisontine rassemblait alors près de 400 fidèles au culte dominical. Nous avons poursuivi nos investigations à Besançon jusqu'en 2005, dans le cadre de notre thèse de doctorat.

3. Nous avons mené des investigations à la Mission du Plein Évangile. La Porte Ouverte chrétienne entre 2002 et 2004, en collaboration avec Jean-Paul Willaime dans le cadre d'une recherche subventionnée par la Fédération protestante de France. Cette assemblée, généralement considérée comme une des plus importantes Églises pentecôtistes de France, rassemblait au moment de l'étude environ 1300 fidèles au culte dominical (Willaime et Amiotte-Suchet 2004). Voir aussi Fath (2008, 151-153).

4. La Fédération Évangélique Missionnaire (FEM) a été fondée par René Kennel en 1989 pour fédérer l'énergie de l'ensemble des assemblées nées sous son ministère. 
douloureuses par le passé ${ }^{5}$, avait décidé de limiter au minimum la création d'Églises-filles ${ }^{6}$ et d'encourager ses fidèles à parcourir chaque semaine de nombreux kilomètres afin que tous se retrouvent dans la méga-Église de Mulhouse. Prenant de l'âge, les deux hommes avaient progressivement confié le flambeau à leur fils — Étienne Kennel et Samuel Peterschmitt — afin que leurs œuvres continuent de se développer. Mais l'un et l'autre conservaient au sein de leurs assemblées respectives un charisme personnel intact, tant ils étaient pour les membres des assemblées des «hommes de Dieu» dont l'histoire personnelle, faite d'épreuves et d'intégrité, constituait un modèle d'exemplarité, une preuve en soi de l'authenticité de la mission que Dieu leur a confiée 7 .

Comme le rappellent André Corten et André Mary, l'origine précise du pentecôtisme demeure floue puisque si

pour certains, elle remonte aux mouvements de réveil du Tenessee de 1886 desquels est issue en 1907 la Church of God (Cleveland); pour d'autres, le pentecôtisme naît en janvier 1901 dans la communauté blanche de Topeka (Kansas) animée par le pasteur méthodiste Charles F. Parham [...]. De plus en plus - lorsque l'on veut souligner la composante "africaine » du pentecôtisme -, on retient la date de 1906 marquée par l'effervescence religieuse d'une communauté noire de Los Angeles (Azusa Street Mission du pasteur baptiste William J. Seymour) qui se répand rapidement un peu partout dans le monde et notamment en Amérique latine et en Afrique. (Corten et Mary $2000,11)^{8}$

5. Avant 1984, la POC n'était pas une œuvre indépendante et Jean Peterschmitt devait composer avec un collège de pasteurs et d'anciens. C'est en 1984 que plusieurs de ces pasteurs ont souhaité prendre plus d'autonomie vis-à-vis de la tutelle que faisait peser Jean Peterschmitt sur le groupe. Ce dernier s'est opposé à cette prise d'autonomie et, devant la détermination des autres pasteurs, a dû accepter à contrecœur de voir son œuvre se diviser. À la suite de cette expérience douloureuse, Jean Peterschmitt n'a plus favorisé la naissance d'Églises annexes et préféré la forme d'une méga-Église à Mulhouse.

6. On appelle Églises-filles (ou Églises-annexes) les assemblées demeurant sous la tutelle d'une Église-mère.

7. Pour un récit détaillé des histoires respectives de ces deux assemblées pentecôtistes de l'Est de la France, voir notre thèse de doctorat (Amiotte-Suchet 2006, 697-730).

8. Pour la majorité des pentecôtistes, 1906 constitue la date fondatrice, les événements précédents étant considérés comme des moments précurseurs, même s'il est toujours difficile de distinguer chronologiquement les racines du holiness movement et le grand réveil de 1906 (voir Brandt-Bessire 1986). 
Reste que la principale caractéristique du pentecôtisme, qui le spécifie dans ses influences méthodistes, revivalistes et fondamentalistes ${ }^{9}$, réside dans sa compréhension littéraliste du second chapitre des Actes des apôtres. Pour les porteurs du réveil pentecôtiste, le $\mathrm{Xx}^{\mathrm{e}}$ siècle est le temps de la réactualisation des charismes de l'Église primitive. Les dons de l'Esprit que Dieu avait fait descendre sur les apôtres au jour de la Pentecôte (parler en langues, dons de prophéties, de visions, de guérisons, etc. ${ }^{10}$ ) avaient pendant des siècles disparus en raison des égarements de l'Église chrétienne. Mais pour les pentecôtistes, ces dons sont à nouveau disponibles aux convertis. Cette "pluie de l'Esprit ", sensée se répandre sur les fidèles lorsqu'ils sont réunis, constitue autant une confirmation divine de la conformité doctrinale des assemblées qui en sont pourvues qu'un signe prémonitoire de l'imminente seconde venue du Christ.

Bref, les pentecôtistes vivent dans un monde réenchanté où la main de Dieu — tout comme celle de Satan — est omniprésente et où les miracles et les tentations malignes se côtoient au quotidien. Les temps qu'ils croient vivre correspondent terme à terme à ceux qui sont décrits dans la Bible, ces premiers temps qui ont suivi la naissance du Christ où l'effusion de l'Esprit saint unissait les apôtres. Ils attendent de leur conversion la réalisation du millénium, ce qui les couvrirait de biens matériels et spirituels et constituerait un véritable accomplissement personnel ici-bas, comme dans l'au-delà. (Droz 2002, 89)

Si cette sensibilité spécifique du protestantisme évangélique prend ses origines au début du $\mathrm{xx}^{\mathrm{e}}$ siècle, elle va considérablement se répandre et se diversifier à partir des années 1970 sous sa forme dénominationnelle, tout en se répandant également au sein des assemblées évangéliques comme à l'intérieur des Églises catholique et protestante réformée dans ce qu'il est convenu d'appeler le mouvement charismatique ${ }^{11}$. Du pentecôtisme dit historique qui va commencer de s'implanter en France dans les années 1930

9. Sur les différents «moments » de l'histoire du protestantisme évangélique, on pourra se reporter à la synthèse très éclairante de Christopher Sinclair (2002).

10. «Or, à chacun la manifestation de l'Esprit est donnée pour l'utilité commune. En effet, à l'un est donnée par l'Esprit une parole de sagesse; à un autre, une parole de connaissance, selon le même Esprit; à un autre, la foi, par le même Esprit; à un autre, le don des guérisons, par le même Esprit; à un autre, le don d'opérer des miracles; à un autre, la prophétie; à un autre, le discernement des esprits; à un autre, la diversité des langues; à un autre, l'interprétation des langues. Un seul et même Esprit opère toutes ces choses, les distribuant à chacun en particulier comme il veut » (1 Co 12,7-11).

11. Sur la diffusion transnationale du pentecôtisme, voir Martin (2002), Corten et Mary (2000), Corten (2006). 
sous sa forme Assemblée de Dieu (ADD), au Renouveau charismatique catholique qui va naître dans les années 1970, en passant par toutes les dénominations pentecôtistes nouvelles et les influences charismatiques qui vont reconfigurer certaines dénominations protestantes évangéliques existantes, la plus grande diversité règne au sein de cette nébuleuse qui s'inscrit dans la continuité de la mouvance revivaliste (Pierre-Louis Lavigne 2003).

Les deux assemblées pentecôtistes sur lesquelles porte ce texte sont donc nées des $\mathrm{ADD}$, mais leurs pasteurs ont choisi la voie de la rupture pour développer leurs œuvres respectives en toute autonomie ${ }^{12}$. Dans les récits de conversion qui nous furent donnés par les fidèles, l'expérience intime de la présence de Dieu, à travers l'Esprit saint, y était toujours centrale. Car si la conversion est d'abord pensée et publiquement valorisée comme un choix personnel opéré à l'âge adulte ${ }^{13}$ par celui qui décide de «s'engager avec Christ », elle ne saurait pour eux s'avérer " authentique » en l'absence d'une expérimentation personnelle de la puissance divine. En effet, si ce choix personnel s'officialise publiquement par «l'entrée dans les eaux du baptême ", c'est-à-dire la participation au rituel du baptême par immersion qui marque officiellement l'entrée du chrétien au sein de la communauté des convertis, c'est bien le fait de faire l'expérience personnelle de la présence divine (et d'acquérir par là même la certitude du salut) qui est essentielle et fait du converti un chrétien né de nouveau (born again). Dans cette démarche, le «baptême en Esprit » (se traduisant généralement par le parler en langues) constitue par les fidèles des assemblées étudiées une seconde expérience qui va confirmer l'authenticité de la conversion, même si dans les récits de vie le moment de l'expérience de la présence divine et celui du «baptême en Esprit» peuvent tout à fait se confondre. Si le mouvement méthodiste fondé par John Wesley au XVIII ${ }^{\mathrm{e}}$ siècle en Angleterre avait initié cette valorisation de l'expérience d'un baptême "dans l'Esprit » comme preuve de l'authenticité du baptême d'eau, le pentecôtisme a ceci de particulier qu'il va faire de cette expérience extra-ordinaire une expérience de tous les jours par la pratique collective du «parler en langues» (ou glossolalie $^{14}$ ), une louange linguistiquement incompréhensible qui, au sein de ces

12. Pour mieux situer la POC dans son environnement, on pourra se reporter utilement à l'ouvrage de Raymond Pfister (1995) sur l'implantation du pentecôtisme en Alsace.

13. Le pentecôtisme s'inscrit pleinement dans la mouvance protestante évangélique régulièrement qualifiée de "christianisme de conversion ». Voir notamment l'ouvrage collectif sous la direction de Sébastien Fath (2004).

14. Voir l'ouvrage de Nathalie Dubleumortier (1997), ainsi que la définition de la notion de «glossolalie» donnée par Patrick Sbalchiero (2002). 
assemblées, est pensée comme la conséquence directe de l'irruption de l'Esprit saint dans le corps des convertis, comme la «reconfirmation » quasi quotidienne de l'authenticité de la conversion, mais aussi comme le rappel des promesses faites par Dieu à tous ceux qui ont choisi de répondre à son invitation. S'il convient donc de donner («accepter Christ dans son cœur») pour recevoir ( «expérimenter la présence de Dieu»), il convient également de rendre («s'engager à suivre Christ») pour recevoir à nouveau («acquérir la puissance divine»). Dans ce cercle infini d'échanges, l'important est toujours de "rester en ligne ${ }^{15}$ ", de garder le contact avec Dieu dans chaque moment de son existence. Parler en langues permet de prouver publiquement que l'on entretient, comme les autres, un contact direct avec Dieu. Dans cette configuration, la question de l'individu dans la relation religieuse se trouve ainsi posée. Si nul ne saurait naître chrétien, puisqu'il convient d'opérer le choix personnel de "s'engager avec Christ", nul ne saurait non plus considérer que sa conversion est réalisée une fois pour toute, puisqu'il convient chaque jour et à chaque instant, disent-ils, de (re)vivre «l'expérience de l'Esprit».

\section{La relation à Dieu, une intimité toujours mise à l'épreuve}

Dans la continuité d'un mouvement de dé-médiatisation ${ }^{16}$ impulsé dès les premières heures de la Réforme, la mouvance charismatique valorise, dans son discours, l'impératif d'une relation intime, immédiate et sensible entre l'homme et son Créateur. En mettant ainsi l'accent sur l'expérience personnelle de l'immédiateté de l'agir divin, les assemblées pentecôtistescharismatiques secondarisent l'exégèse biblique (et la formation universitaire du pasteur) au profit d'une théologie de l'inspiration personnelle. «Avoir fait l'expérience de l'Esprit » s'impose ainsi à tous comme le premier critère de validité de la conversion et l'art de témoigner publiquement de ses expériences constitue une performance très valorisée au sein des assemblées. Tout se passe comme si rien ne comptait plus que l'expérience personnelle de la «présence », constituant en elle-même la "preuve » du bien-fondé de la démarche de conversion.

15. Expression pentecôtiste reprise par Yannick Fer pour illustrer ce qu'il appelle un «évangile relationnel » (Fer 2005, 155).

16. Au sens où toute l'histoire du protestantisme, depuis les réformes magistérielles et radicales du $\mathrm{XVI}^{\mathrm{e}}$ siècle, est traversée par la valorisation d'un retour aux Écritures et le rejet d'un modèle catholique institutionnel-rituel qui sacralise l'institution et fait du prêtre et des rites les médiations incontournables de la mise en présence du divin. 
Bien des récits de conversion portent la marque de la démarche expérimentale. Faire une expérience, puis deux, puis trois... réagir et se questionner, commencer d'envisager les Écritures autrement, «mettre Dieu à l'épreuve » et faire de nouvelles expériences, relire alors les Évangiles pour y chercher des réponses, ressentir la présence de Dieu et l'appeler à se manifester à nouveau dans sa vie, voir des situations désespérées se résoudre brutalement, capituler devant l'évidence et « donner son cœur à Jésus »... Voilà de quoi sont faits ces récits de conversion, cherchant à mettre en évidence, dans le cheminement sinusoïdale qu'ils mobilisent pour se structurer, la démarche pragmatique qui aura caractérisé le temps du questionnement (Amiotte-Suchet 2006, 269-277).

Pour ma première expérience, ça ne m'emballait pas trop parce que je refusais un peu. J'écoutais les gens témoigner et je me disais: "Mais mon pauvre vieux, qu'est-ce que tu fais là ?» [...] Et puis finalement j'ai cédé, entre guillemets, au fait de faire au moins une expérience avec le Seigneur. Je souffrais depuis pas mal d'années de traumatismes psychologiques, j'étais suivi par un psy tous les quinze jours et il me donnait des cachets pour dormir. [...] Alors je me suis approché à l'imposition des mains et j'ai demandé à ne plus avoir à prendre ces médicaments, à ne plus souffrir d'angoisses. Et je peux te dire que le Seigneur a vraiment agi [...]. J'avais cette conviction que si j'allais me coucher, je dormirais, et j'ai dormi comme un bébé. Aujourd'hui, je n'ai plus de cachets pour dormir! (Stéphane, EEP)

Et j'ai fait plusieurs expériences de ce côté-là, comme à un moment donné j'étais assis dans mon lit et je me posais une question, j'ouvre ma Bible en étant perdu dans mes pensées et le premier verset sur lequel je tombe était la réponse précise, comme un flash porté au visage, la réponse à la question qui triturait mon esprit. Mais c'était une révélation pour moi. Peut-être un verset que j'avais déjà lu, que je pourrais lire maintenant et qui ne me touche plus, mais à ce moment-là, c'était absolument la réponse claire, transparente, précise. (Albert, POC)

[...] et ce soir-là dans mon cœur, je luttais, je ne voulais pas croire en Dieu, et puis en même temps je savais bien qu'il y avait quelque chose et, au fond de moi-même, j'ai dit à Dieu, de tout mon cœur, j’ai dit : "Seigneur, je suis prêt à te suivre ! » Et j'ai été visité par Dieu à ce moment, tout simplement ! » (un fidèle lors d'un témoignage public à l'EEP)

Cet attachement à mettre en récit le temps du doute et de la mise à l'épreuve de la vérité des Écritures participe de la construction publique de 
la véracité du témoignage. Très critiques vis-à-vis du modèle institutionnel-rituel de l'Église catholique, les assemblées étudiées cherchent pardessus tout à valoriser publiquement la démarche expérimentale, parce qu'elle constitue pour elles l'exact opposé des identités religieuses héritées. La conversion est ainsi décrite comme un processus dont le principe fut (et demeure) de tester la réalité des promesses avant de s'engager à changer de vie pour profiter durablement des bienfaits de la vie chrétienne.

Moi je le dis souvent quand je témoigne, si j'avais trouvé dans l'assemblée une vie de religion, de religieux, je ne serais jamais venu. Moi je suis venu dans l'assemblée parce qu'il y avait quelque chose de différent, que ne pouvait pas m'apporter une religion. Une vie où Dieu agit concrètement, par son Esprit, par sa Parole, et qui prouve cette Parole par l'accomplissement des promesses qui sont dans cette Parole, un Dieu qui t'assiste tous les jours et qui est avec toi chaque jour. [...] Un Dieu qui vit avec toi les choses que tu vis, qui est là pour te relever quand tu te casses la figure, qui est là pour t'encourager quand ça ne va pas, et qui est là aussi quand tout va bien. [...] Moi je crois que c'est pour cela que je suis chrétien, c'est parce qu'il y a une vie, quelque chose de vivant, parce qu'il y a des assurances, des convictions que Dieu nous donne par sa Parole et son Esprit! (Stéphane, EEP)

Dans les mises en récit, le vécu physiologique de la présence de Dieu est une expérience récurrente qui s'égraine de l'appel à l'engagement pour progressivement devenir une expérimentation quasi quotidienne. Elle est décrite comme une "récompense » gratifiant le nouveau venu pour chaque pas effectué dans la bonne direction. Preuve irréfutable, l'expérimentation personnelle de l'existence de Dieu est non seulement au fondement de la démarche de conversion, mais elle est également décrite comme l'un des meilleurs « remparts » contre les mises en doute du monde extérieur. Le modèle narratif de la conversion met ainsi en scène un nouveau venu qui passe une période plus ou moins longue de sa conversion à " poser des toisons ", c'est-à-dire en somme à mettre Dieu à l'épreuve des faits pour se constituer un «stock» d'expérimentations personnelles suffisamment important pour que ses certitudes ne soient plus remises en cause. Reconstruites et exemplarisées pour s'intégrer dans le récit de conversion, ces expériences seront l'objet d'incessantes narrations, lors de soirées d'évangélisation, d'évangélisation de rue, de conversations ordinaires ou d'entretiens avec le chercheur.

Cette nécessité de la preuve par l'expérience ne va bien évidemment pas sans poser un certain nombre de difficultés, en particulier quand le malheur et la maladie persistent. Bien des prises de distances avec l'assem- 
blée commencent, sans doute ${ }^{17}$, par l'incapacité à intégrer une situation personnelle difficile durable dans le schéma narratif de la conversion. Se convertir et faire le récit public de sa conversion sont les deux faces d'une même médaille, car c'est par l'organisation et la stabilisation de différents éléments biographiques dans un récit que l'individu attribue une signification (la conversion) à son histoire et que cette signification dès lors stabilisée agit de manière performative sur sa mémoire comme sur sa manière d'interpréter de nouveaux événements ${ }^{18}$. Implicitement, chacun intègre le fait que l'exercice du témoignage public constitue d'abord et avant tout la participation à l'administration de la preuve collective de l'actualité des charismes et, par extension, la légitimation de l'autorité du pasteur comme guide spirituel choisi par Dieu lui-même pour accompagner chacun dans l'expérimentation de l'immédiateté de l'agir divin.

\section{L'administration communautaire de la preuve personnelle}

Dans le récit de conversion, la certitude d'avoir opéré le bon choix repose donc d'abord et avant tout sur une expérimentation personnelle et tangible de la puissance divine. Le converti se présente toujours comme conscient et responsable d'un choix de vie qui repose sur sa relation intime et durable avec son Créateur, à travers le canal énergétique que représente l'Esprit saint. Et puisque "choisir de suivre Christ» s'accompagne d'un certain renoncement aux "plaisirs de ce monde " pour consacrer sa vie à la prière et à l'évangélisation du monde, le nouveau venu découvre non sans surprise qu'il peut aussi se montrer exigeant dans cette relation pour obtenir en retour de son engagement les bénéfices de l'expérience des charismes.

Un jour, j'étais assis dans mon lit, je lisais la Bible et je parle à Dieu. Je fais cette prière: "Si tu existes, si tu es ce Dieu de la Bible, si tu es vivant, tu es capable de nous parler, alors donne-moi le Saint Esprit, donne-moi le parler en langues ». [...] C'était samedi ou dimanche, personne ne le savait mais le mardi suivant, à la réunion à Pfastatt, le pasteur donne une prophétie, d'abord le parler en langues, il donne l'interprétation du parler en langues: «Tu m'as prié, tu m'as dit ça, ça, ça!» Il a répondu texto à la prière que

17. N'ayant pas réalisé d'entretien avec des personnes ayant récemment quitté les assemblées étudiées, nous ne pouvons ici que supposer que cette dimension entre éventuellement en ligne de compte.

18. Sur ce point, voir les travaux de Paul Ricœur (1983) concernant la mise en intrigue du récit et ceux de Peter Stromberg (1993) concernant la dimension performative du récit de conversion en milieu évangélique. 
j'avais faite. Quand il a dit ça, j'étais déjà assis mais je suis tombé sur mon derrière et, chose intéressante, elle [ma femme] qui n'était au courant de rien, au moment où il y a la prophétie, elle m'a dit: «Ça, c'est pour toi! » Elle a senti elle-même de nouveau cette onction qui était venue sur nous, elle a ressenti quelque chose dans son cœur, comme une visitation à ce moment-là. [...] J'étais tout heureux et tout excité en disant: "C'est dingue, c'est pas possible, Il est quand même vivant, Il a entendu ma prière ", et j'essayais de comprendre que moi, assis dans mon lit, Dieu m'avait entendu. (Albert, POC)

Cette façon, dans l'intimité de la chambre, de mettre Dieu à l'épreuve en exigeant des preuves de sa présence agissante insiste sur la notion de relation personnelle, indépendante de l'activité communautaire. Et dans le même temps, c'est bien ici durant le culte que la «preuve» est donnée, par l'intermédiaire du pasteur d'abord, qui reçoit, transmet puis interprète la réponse divine, puis de l'épouse du fidèle qui confirme, par son ressenti personnel, l'identité du destinataire. Cet extrait d'entretien, qui n'échappe pas aux reconstructions narratives de tout témoignage de conversion, constitue une excellente illustration de la façon dont l'idée d'une relation immédiate entre l'individu et Dieu s'inscrit dans un processus communautaire d'administration de la preuve. Car si l'individu fait reposer ses certitudes sur l'affirmation publique selon laquelle il vit lui aussi, comme les autres convertis, l'expérience régulière de la visitation de l'Esprit de Dieu, il met en évidence, dans le même temps, que c'est par les autres, et son pasteur en premier lieu, que la preuve de l'authenticité de ses expériences lui est donnée. C'est là le principe de ce que Danièle Hervieu-Léger a défini comme le mode de validation communautaire du croire, où c'est bien la mise en cohérence de ce que vivent et expriment publiquement les différents membres de la communauté qui constitue la source de légitimation du croire communautaire (Hervieur-Léger 1999).

"Ce matin », " aujourd'hui », "présent ", "maintenant ", " en cet instant » sont sans aucun doute, avec "Dieu ", "Jésus » et "Seigneur », les termes les plus récurrents dans la bouche des pasteurs tout au long du culte dominical. Des prières d'ouverture au chant final, l'ensemble du culte apparaît comme un enchaînement méticuleux de séquences visant à mettre en scène l'irruption progressive (et d'intensité croissante) de l'Esprit saint sur l'assemblée des fidèles. Le souci permanent des pasteurs est d'éviter la routinisation des activités collectives qui transformerait, selon eux, le culte en un simple «rituel ». La réunion publique doit en somme être chaque semaine présentée, construite et animée comme une réunion d'exception, un jour pas 
comme les autres où quelque chose est censé se jouer, ici et maintenant, pour tous ceux qui ont franchi la porte de la salle. «Dieu t'appelle ce matin! Dieu a un grand projet pour toi ce matin! » C'est par ce type de formules personnelles que les pasteurs interpellent les individualités réunies avant de s'adresser directement à Dieu pour l'inviter à répandre son Esprit sur l'assemblée. Et très vite la preuve en est donnée, par les prophéties et paroles de connaissance qui font irruption grâce aux pasteurs et porteurs de charisme, assurant la "mise en présence du divin" (Amiotte-Suchet 2006). C'est alors bien Dieu qui parle, promettant l'amélioration des conditions d'existence à ceux qui suivront sa parole.

Pendant qu'il nous renouvelle, mettez la main là où vous souffrez, vous avez besoin de l'aide de Dieu, mettez la main à l'endroit où vous souffrez, et recevez maintenant la guérison! [...] Une joie ineffable que vous ne connaissiez pas vous envahit ce matin, maintenant, en cet instant! (Samuel Peterschmitt ${ }^{19}$ )

[...] le Seigneur te dit: «Cherche ma face, redonne-moi une place dans ta vie et surtout... réagis selon ma Parole, sois vraiment source de paix autour de toi, et tu verras, je changerai, là où tu es, je transformerai la situation où tu te trouves ${ }^{20}$ !»

Soyez positif par les paroles de votre bouche, et vous verrez combien je vous ai placés sur un chemin de victoire. Car c'est moi qui vous restaure, c'est moi qui vous donne la joie de vivre, c'est moi qui vous donne la réussite. J'ai payé à la croix, j'ai tout accompli afin que vous soyez vraiment des enfants heureux, des enfants qui en toutes circonstances soient remplis d'action de grâce. [...] Car le chemin, c'est moi votre Seigneur qui est le chemin, la vérité et la vie ! Et je suis fidèle dans mes promesses dit le Seigneur l'éternel Dieu! » (Jean Peterschmitt ${ }^{21}$ )

il y a un frère parmi nous qui souffre de la prostate mais le Seigneur lui dit: «Sache mon enfant que ce matin je pose ma main bénissante et guérissante sur toi ${ }^{22}$.»

L'articulation permanente entre le «il vous dit» et le «je vous dis» brouille l'identité du locuteur et la temporalité de la parole délivrée, au point que nul ne sait vraiment très bien qui parle et à quel moment le message a

19. Culte du $1^{\text {er }}$ décembre 2002.

20. Parole de connaissance d'une fidèle de la POC lors du culte du 06 octobre 2002.

21. Prophétie donnée au cours du culte du 26 mai 2002.

22. Parole de connaissance donnée par un fidèle de la POC lors du culte du 26 mai 2002. 
été transmis. À l'assemblée mulhousienne, les porteurs d'un charisme de prophétie sont amenés à faire la queue derrière le micro pour délivrer une prophétie ou une parole de connaissance, mais au moment où ils prennent la parole, leur mode d'élocution cherche à mettre en scène la transmission d'un message qui leur est dicté "en direct». La présence immédiate de l'Esprit saint se trouve ainsi objectivée publiquement par la pratique des charismes où s'enchaînent des encouragements collectifs et des promesses de guérison individuelles, des messages pour tous formulés comme des messages pour chacun. Cette exercice collectif de mise en présence prépare et donne sa légitimité à la prédication du pasteur, qui n'est pas pensée comme un enseignement issu des réflexions personnelles de ce dernier mais bien comme une prestation orale d'inspiration divine dont l'objectif est d'interpeller personnellement chacun sur un point particulier de son existence.

À ce moment-là, le pasteur, quand il prêchait, il dévoilait toute ma vie, il montrait tout ce que j'avais besoin, il montrait les manques que j'avais dans mon cœur, et je me disais: Mais cet homme-là, il connaît ma vie. " (un pasteur de la Fédération Évangélique Missionnaire témoignant lors d'une pastorale)

Chaque fois que je vais aller à la réunion, il y a toujours quelque chose pour moi qui va venir de la Parole. Mais il y a des fois, je vais être préoccupé et puis je ne vais pas me laisser prendre par la Parole. Tu vois? Je vais rien recevoir. » (Pascal, EEP)

La prédication valorise bien évidemment le recours indispensable aux Écritures, mais dans la logique d'une lecture inspirée. C'est le chrétien qui lit mais c'est bien Dieu qui lui montre ce qu'il doit lire et qui guide sa compréhension immédiate d'un passage qui prend d'abord sens ici et maintenant, en référence à sa situation présente. Dans une prestation orale qui peut parfois durer jusqu'à 60 minutes, le pasteur s'attache à mettre en cohérence des anecdotes troublantes de son expérience pastorale avec la signification des passages que Dieu lui a mis à cœur ce dimanche matin. L'objectif consiste ici à mettre en évidence l'actualité des Écritures, et le pasteur mobilise dans cet exercice autant de témoignages prouvant que le Seigneur sait effectivement tenir ses promesses envers ceux qui gardent fermement le cap de leur engagement. La réunion publique peut ainsi s'achever par l'exercice collectif du parler en langues, où la présence immédiate de l'Esprit se trouve collectivement objectivée par ces "paroles » pensées comme les conséquences linguistiques de la visitation de l'Esprit dans le corps de tous les convertis. Qu'il soit long ou bref, le moment où toute la communauté se met à par- 
ler en langues apparaît comme une séquence indispensable sans quoi la preuve de l'irruption de l'Esprit de Dieu au sein de la communauté des fidèles ne serait pas totalement fournie. Les expressions corporelles associées à la pratique des langues cherchent à traduire par la gestualité et le rythme des élocutions une certaine perte d'emprise du converti sur son corps, mettant ainsi en évidence la puissance d'une expérience intérieure si forte qu'elle bousculerait brièvement la maîtrise sociale des émotions. Le recours au registre émotionnel dans le cadre des célébrations constitue ici l'objectivation d'une expérience à la fois personnelle et communautaire de l'aboutissement réussi de la réunion des fidèles, et par là même de la compétence du pasteur à faire advenir ce moment si indispensable à la collectivisation des expériences individuelles. Si, comme le faisait remarquer Patrick Williams à propos du pentecôtisme tsigane, le parler en langues, par certains côtés, constitue donc une démocratie de l'expression ${ }^{23}$, une démocratie de l'accès au divin objectivant publiquement l'immédiateté de la relation entre l'individu et son Créateur, cet accès demeure néanmoins médiatisé par le pasteur et les porteurs d'un charisme de prophétie qui apparaissent ici comme des fils conducteurs indispensables à l'établissement de cette relation personnelle ${ }^{24}$. Si la pratique des langues peut donc être lue comme l'objectivation d'une relation intime entre le fidèle et son Dieu, elle constitue aussi, dans l'économie de la réunion, une preuve collective de la qualité de la prestation pastorale.

\section{Conclusion}

En pentecôtisme, cette valorisation d'une relation interpersonnelle entre l'individu et son Créateur est l'objet d'un travail de recadrage constant par le collectif, en particulier par le biais des pasteurs et des anciens qui agissent comme les matrices des expressions publiques en recadrant constamment les gestuelles et les énonciations et en donnant le «feu vert» aux

23. «La parole appartient à tous (tous sont invités à «témoigner », voire à se faire exégète et à enseigner), tous ont accès aux événements et expériences les plus profondes de la vie religieuse : parler en langues, don de guérison...» (Williams 1991, 95).

24. Sur le rôle du pasteur dans le déroulement de la célébration en milieu pentecôtiste, voir aussi Amiotte-Suchet (2008).

25. Yannick Fer a particulièrement bien mis en évidence, à partir de ses travaux sur les Assemblées de Dieu en Polynésie française, le rôle central des pasteurs et des anciens dans ce qu'il appelle des "dispositifs "invisibles" de socialisation, de formation et de contrôle communautaire" (Fer 2005, 153). 
manifestations charismatiques ${ }^{25}$. Il est ici approprié de qualifier le pentecôtisme d' "individualisme communautaire ", parce le modèle cultuel que ces assemblées mobilisent permet d'objectiver pour chacun que ce qu'il vit et exprime avec l'Esprit saint est bien conforme (et donc "authentique») à ce que les autres ressentent et manifestent. La validation est donc à la fois mutuelle et communautaire puisque le collectif ne permet pas seulement à chacun de trouver en l'autre la confirmation de l'«authenticité » de ce qu'il vit, il permet surtout de construire l'idée que ces expériences individuelles " authentiques » ne peuvent être réalisées que grâce à la stimulation de l'expérience collective des réunions de prières orchestrées par les pasteurs. Ceux qui se rendent chaque semaine à l'assemblée viennent donc pour participer à la «mise en présence du divin » en étant des agents, par leur corps, de cette présence. En participant de la sorte, ils reconfirment aux autres (et à Dieu) la sincérité de leur engagement tout en espérant chaque fois recevoir des récompenses immédiates pour leur pugnacité à "suivre Christ». Les assemblées sur lesquelles ont porté nos investigations ethnographiques ne valorisent pas un automatisme des bienfaits tel qu'il peut être pratiqué dans des mouvements pentecôtistes comme l'Église Universelle du Royaume de Dieu où la théologie de la prospérité pousse un cran plus loin cette logique du donnant, donnant en associant réussite sociale et qualité de la foi (voir Aubrée 2000; Corten, Dozon et Oro 2003). Ceux que nous avons côtoyés à Besançon ou à Mulhouse ne reconnaissent pas dans les signes extérieurs de richesse la qualité d'une conversion. L'infortune prolongée demeure toujours une situation complexe à interpréter et ne se résume pas aux simples conséquences d'un manque de foi et de persévérance dans la prière. Mais le scénario propre aux réunions des assemblées est là pour faire oublier le passé et renforcer la conviction que c'est ici, maintenant, que tout va basculer. L'immédiateté du contact avec le divin est collectivement construite par ces médiateurs que sont les pasteurs et les porteurs de charismes, qui préparent, légitiment et orchestrent la «spontanéité » du parler en langues et assurent par là même la mise en présence du divin.

\section{Références}

Aмiotte-Suchet, L. (2006), Pratiques pentecôtistes et dévotion mariale : analyse comparée des modes de mise en présence du divin, thèse de doctorat inédite, Paris, École Pratique des Hautes Études.

(2008), «Il pastore pentecostale : un filo conduttore tra il cielo e la terra ", Etnografia, 1, p. 45-63. 
Aubree, M. (2000), "La diffusion du pentecôtisme brésilien en France et en Europe : le cas de l'I.U.R.D.", dans C. Lerat et B. Rigal-Cellard, dir., Les mutations transatlantiques des religions, Bordeaux, PUB, p. 149-157.

BrandT-Bessire, D. (1986), Aux sources de la spiritualité pentecôtiste, Genève, Labor et Fides.

Corten, A., J.-P. Dozon, et A. Oro, (2003), dir., Les nouveaux conquérants de la foi : l'Église Universelle du Royaume de Dieu, Brésil, Paris, Karthala.

Corten, A. et A. MARY (2000), «Introduction », dans A. Corten et A. Mary, dir., Imaginaires politiques et pentecôtismes, Paris, Karthala.

CORTEN, A. (2006), «Un religieux immanent et transnational », Archives de sciences sociales des religions, 133, p. 135-151.

Droz, Y. (2002), «Esquisse d'une anthropologie de la conversion; pratiques religieuses et organisation sociale en pays kikuyu ", dans R. Massé et J. Benoist, dir., Convocations thérapeutiques du sacré, Paris, Karthala.

Dubleumortier, N. (1997), Glossolalie: Discours de la croyance dans un culte pentecôtiste, Paris, L'Harmattan.

FATH, S. (2004), dir., Le protestantisme évangélique, un christianisme de conversion, Turnhout, Brepols.

FATH, S. (2008), Dieu XXL. La révolution des megachurches, Paris, Autrement.

FER, Y. (2005), "Genèse des émotions au sein des assemblées de Dieu polynésiennes ", Archives de sciences sociales des religions, 131-132, p. $143-163$.

HeRvieu-LÉGER, D. (1999), Le pèlerin et le converti. La religion en mouvement, Paris, Flammarion.

Martin, D. (2002), Pentecostalism: The World their Parish, Oxford, Blackwell.

PFister, R. (1995), Soixante ans de pentecôtisme en Alsace (1930-1990), Frankfurt, Peter Lang.

Pierre-Louis Lavigne, T. (2003), «Essai de typologie des pentecôtismes français ", Bulletin de la Société de l'Histoire du Protestantisme Français, 149, p. 99-126.

Ricceur, P. (1983), Temps et récit. Tome I: L'intrigue et le récit historique, Paris, Seuil. 
Sbalchiero, P. (2002), "Glossolalie ", dans P. Sbalchiero, dir., Dictionnaire des miracles et de l'extraordinaire chrétiens, Paris, Fayard, p. 318-320.

Sinclair, C. (2002), "Introduction: définition et historique ", dans

C. Sinclair, dir., Actualité des protestantismes évangéliques, Strasbourg, PUS, p. 7-25.

Stromberg, P. G. (1993), Language and Self-transformation. A Study of the Christian Conversion Narrative, Cambridge, Cambridge University Press.

Willaime, J.-P. et L. AmiotTe-Suchet (2004), La pluie de l'Esprit. Étude sociologique d'une assemblée pentecôtiste mulbousienne. Rapport de recherche subventionné par la Fédération protestante de France, octobre 2004, texte disponible sur HAL-SHS: $<$ http://halshs.archives-ouvertes.fr/halshs-00124902>

Williams, P. (1991), «Le miracle et la nécessité: À propos du développement du pentecôtisme chez les Tsiganes ", Archives de sciences sociales des Religions, 73, p. 81-98.

\section{Résumé}

Lorsqu'il est amené à témoigner de sa conversion, le membre d'une assemblée pentecôtiste insiste avant tout sur la dimension personnelle de son choix de vie et sur les manifestations divines qui l'ont encouragé et qui l'encouragent encore chaque jour dans cette voie. Avoir fait l'expérience personnelle de l'agir divin est un élément constitutif de tout récit de conversion. Cet article, basé sur des recherches doctorales menées auprès de deux assemblées pentecôtistes de l'Est de la France, cherche à mettre en évidence que cette relation personnelle et intime entre le fidèle et son Créateur se construit dans le cadre communautaire : à travers l'harmonisation des récits de conversion et la structuration des réunions de prières assurant par les manifestations charismatiques la mise en présence de l'Esprit saint au sein de la communauté des fidèles. C'est en effet sur une validation communautaire que reposent en partie les convictions individuelles.

\section{Abstract}

When providing testimony about his conversion, the member of a Pentecostal assembly insists above all on the personal dimension of his choice of life and on the divine manifestations which encouraged him and continue 
to support him along the way. The personal experience of God's action is a constituent element of any narrative of conversion. This article, based on my PhD field research with two Pentecostal assemblies located in the east of France, highlights the role of the community in constructing this personal and intimate relation between the believer and his creator through the harmonization of the narratives of conversion and structuring of prayer meetings which ensure, by way of charismatic manifestations, the Holy Spirit's presence in the midst of the community of believers. Individual beliefs are thus partly based on substantiation by the community. 